\title{
Feasibility Study on the Microspheres for Deep Profile Control Technology in High Permeability of High Temperature and High Salinity Reservoirs
}

\author{
Changchun $\mathrm{Y}^{1,2 *}$, Yue Xiangan ${ }^{1,2}$, Zhou Daiyu ${ }^{3}, \mathrm{Xu} \mathrm{Rui}^{1,2}$, Zhao $\mathrm{Ji}^{3}$, Li Chaoyue ${ }^{1,2}$ and Zhang Xuenan ${ }^{1,2}$ \\ ${ }^{1}$ Key Laboratory of Petroleum Engineering, Ministry of Education, China University of Petroleum, Beijing 102249, P.R. of China \\ ${ }^{2}$ College of Petroleum Engineering, China University of Petroleum, Beijing 102249, P.R. of China \\ ${ }^{3}$ Tarim Oilfield Branch Company, PetroChina, Kuerle, Xinjiang 841000, P.R. of China
}

\begin{abstract}
In order to obtain polymer microsphere for profile control and water shutoff with good suspension, high temperature resistance and high salinity tolerance, micrometer DCA (divinylbenzene-co-acrylamide) microsphere which had a three-layer structure was synthesized by the monomer of acrylamide, initiator of azobisisobutyronitrile, crosslinking agent of divinylbenzene and emulsifier of ethoxylated lauryl alcohol sulfate. Basic performance of DCA microsphere was evaluated, including temperature resistance, plugging capacity, effect of profile control and water shutoff and method optimization. The results showed that the particle size of DCA microsphere ranged from $2 \mu \mathrm{m}$ to $339 \mu \mathrm{m}$ and the shell had a Nano porous structure. The temperature resistance of DCA microsphere was up to $300^{\circ} \mathrm{C}$. The residual resistance coefficient of each segment along artificial homogeneous cylindrical core was still more than 2 with a strong deep plugging efficiency. After water flooding of oil in two-dimensional heterogeneous cores was carried out, the slug of DCA microspheres was injected. The amplitude of enhanced oil recovery was up to $10.18 \%$ in stage of the subsequent water flooding. On the basis of the subsequent water flooding, the recovery of carbon dioxide flooding was $19.64 \%$. Feasibility methods which were the plugging of DCA microspheres and carbon dioxide flooding on deep profile control technology in high permeability of high temperature and high salinity reservoirs have been proposed.
\end{abstract}

Keywords: Temperature; Salinity reservoir; High permeability; Microspheres; Deep profile control

\section{Introduction}

Profile control and water shutoff are still considered technical problems for high-temperature and high-salinity reservoirs. Given that the conventional agents of profile control and water shutoff have many disadvantages, their performance in terms of high temperature and salinity tolerance is poor. Their cross-linking reaction time is difficult to control, and the validity period of the operation measures is short. A system with high temperature and salinity tolerance should be further studied. In recent years, deep profile control and water shutoff have been implemented in reservoirs using the new technology of the polymer microspheres. The advantage of polymer microspheres includes hightemperature and high-salinity tolerance, good suspension, migrating into the deep reservoir to real-time plugging, the long valid period of the operation measures. At present, deep profile control of polymer microspheres which was used in oilfield mainly was synthesized by the methods of inverse emulsion polymerization [1], inverse suspension polymerization [2] and the dispersion polymerization [3]. According to the mechanism of deep profile control of polymer microspheres, the initial diameter of polymer microspheres which was nanometer or micrometers can be divided into three categories. First category, the expansion of polymer microspheres is mainly through the bridge plugging [4-6]. Second category, the expansion of polymer microspheres interacts with the calcium and magnesium ions to form a network structure to plug $[7,8]$. Third category, the expansion of polymer microspheres adheres to each other to plug. The first category of profile control agent of polymer microspheres was successfully applied in the part of middle or high permeability reservoir in the domestic and achieved good effect of profile control and water shutoff. The microspheres were of the water-swellable type. The microspheres could be easily broken during transport when the multiple series of their expansion was too large. After reaching the deep reservoir, plugging efficiency was significantly reduced. If the multiple expansion was too small, the deformation capacity of the microspheres was poor. Transporting the pore and throat of reservoirs is and was easy to cause pollution and plugging of the reservoir near the injection well. It was not able to achieve deep profile control of the reservoirs $[9,10]$. Microspheres profile control agent entails serious requirements for the reservoir environment. Water formation must contain high calcium and magnesium ions in the second category. In the third category, the external part of the microspheres has a negative charge and can be dissolved in water to swell. The inner layer includes a cross-linked gel with a positive charge. The swelling speed of the inner layer in water is faster than at the outer layer component. Thus, when volume expansion reaches a certain extent, a positive charge is exposed. The microspheres have opposite charges, which occurred the adsorption each other to plug ability. However, such microspheres are easily plugged near well bore area. At the same time, the temperature resistance and good suspension of these three categories of microspheres are poor.

To solve this problem which was high temperature, high salinity and high content of calcium and magnesium ions in Tarim Oilfield, micrometer DCA (divinylbenzene-co-acrylamide) microsphere which had a three-layer structure was synthesized by the monomer of acrylamide, initiator of azobisisobutyronitrile, crosslinking agent of

"Corresponding author: Yang Changchun, College of Petroleum Engineering, China University of Petroleum-Beijing, NO.18 Fuxue Road, Changping, Beijing China, Tel: +86-010-89733960; E-mail: yangchangchun1000@163.com

Received August 03, 2016; Accepted August 30, 2016; Published September 07, 2016

Citation: Changchun Y, Xiangan Y, Daiyu Z, Rui X, Ji Z, et al. (2016) Feasibility Study on the Microspheres for Deep Profile Control Technology in High Permeability of High Temperature and High Salinity Reservoirs. J Pet Environ Biotechnol 7: 296. doi: 10.4172/2157-7463.1000296

Copyright: $\odot 2016$ Changchun $\mathrm{Y}$, et al. This is an open-access article distributed under the terms of the Creative Commons Attribution License, which permits unrestricted use, distribution, and reproduction in any medium, provided the original author and source are credited. 
Citation: Changchun Y, Xiangan Y, Daiyu Z, Rui X, Ji Z, et al. (2016) Feasibility Study on the Microspheres for Deep Profile Control Technology in High Permeability of High Temperature and High Salinity Reservoirs. J Pet Environ Biotechnol 7: 296. doi: 10.4172/2157-7463.1000296

divinylbenzene and emulsifier of ethoxylated lauryl alcohol sulfate [11].

Basic performance of DCA microsphere was studied, including temperature resistance, plugging capacity, effect of profile control and water shutoff and method optimization.

\section{Experimental Procedure}

\section{Chemical reagents}

AM, purchased from Puyang Biological Chemical Co. Ltd, was used as a polymerized monomer. DVB, purchased from Beijing Chemicals Co. Ltd, was used as a crosslinked agent. 2,2-Azobisisobutyronitrile (AIBN), purchased from Puyang Biological Chemical Co. Ltd, was used as initiator. Sodium alcohol ether sulphate (AES), purchased from Beijing Chemicals Co. Ltd, were used as emulsifier. Ethanol was used as solvent. Oil and brine samples were collected from a domestic oilfield. The salinity of simulated water was $269000 \mathrm{mg} / \mathrm{L}$. The core, which was independently developed in a laboratory, was used in the physical simulation experiments.

\section{Equipment}

A DF-101S heat collector and thermostatic heating magnetic stirrer were purchased from HenanYuHua Instrument Co. Ltd. An SC-3610 low-speed centrifuge was purchased from Anhui USTC Zonkia Scientific Instrument Co. Ltd. An FEI Quanta 200 scanning electron microscope was purchased from FEI Co., the Netherlands. An NETZSCH STA409PC thermogravimetric analyzer was purchased from NETZSCH Instruments Manufacturing Co. Ltd, Germany. A Zeiss optical microscope was obtained from Suzhou Matsushita Communication Industrial Co. Ltd. A KQ3200B Ultrasonic Cleaner was purchased from Kunshan Ultrasonic Instrument Co. Ltd. Mastersizer 2000 particle size analyzer was purchased from Malvern Instruments Co. Ltd, UK.

\section{Synthesis of DCA Microspheres}

Emulsion polymerization: A certain amount of AM and AES was dissolved in $100 \mathrm{~mL}$ deionized water to obtain an aqueous phase. A certain amount of AIBN was dissolved in DVB to obtain an oil phase. The oil phase was poured into a $150 \mathrm{~mL}$ beaker. A water bath with constant temperature was made available. The magnetic stirring rate was set to $170 \mathrm{rpm}$. An appropriate amount of aqueous phase was added to the oil phase. After pre-emulsification for certain period of time, the remaining aqueous phase was added to the oil phase. The water temperature was set at a constant $70^{\circ} \mathrm{C}$. After about six hours, the reaction ended. The emulsion was centrifuged at $3500 \mathrm{rpm}$ for 20 minutes to obtain a solid. The solid was washed with ethanol three times and dried for $24 \mathrm{~h}$ in vacuum at $50^{\circ} \mathrm{C}$. White powdery solid DCA microsphere was obtained.

\section{Results and Discussion}

\section{Microstructure and size distribution of DCA microspheres}

The synthetic DCA microspheres were dried by infrared light and were crushed. The photographs of scanning electron microscope (SEM) were shown in Figure 1. The DCA microspheres formulated into the suspension with deionized water. The suspension was placed in an ultrasonic cleaning machine and was dispersed about 5 minutes. The sample was taken with a dropper and was added to 1-liter beaker. The particle size distribution of DCA microspheres was measured by particle size analyzer (Figure 2).

As shown in Figure 2, the particle diameter of DCA microspheres

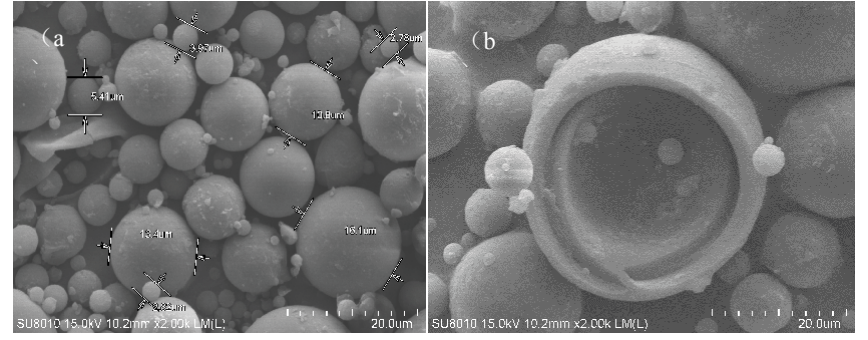

Figure 1: Appearance (a) and internal structure (b) photograph of DCA microspheres.

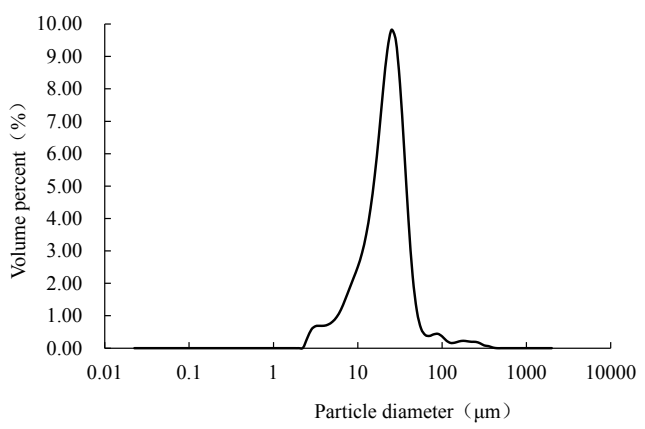

Figure 2: Particle size distribution of DCA microspheres.

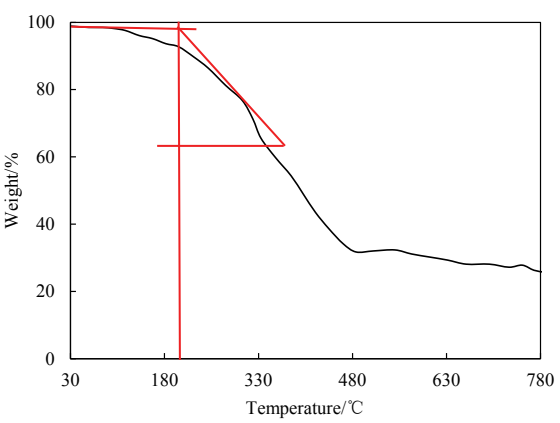

Figure 3: TGA result of HPAM.

range from $2 \mu \mathrm{m}$ to $399 \mu \mathrm{m}$. The inner layer comprises a low boiling point of water. After DCA microspheres were dried, they have a hollow structure. The thickness of the cross-linked polymer layer is $4 \mu \mathrm{m}$. The initial judgment of the DCA microspheres shows that they have better suspension in line with the performance requirements of "injection in reservoir". The shell of the microspheres consists of nanoporous materials. Low water calcium and magnesium ions is injected, migrated into the deep reservoir. DCA Microspheres was injected and migrated into the deep reservoir with water which was the low calcium and magnesium ions. DCA microspheres combined with calcium and magnesium ions of the formation water aggregated into clusters of microspheres which have large enough hydrodynamic radius to plug large pore effectively and achieve microscopic steering of injection water. Structural design can meet above performance requirements. DCA microspheres have a three-layer structure which is the outer layer of the hydration, intermediate layer of cross-linked polymer, inner layer of gel core.

\section{Temperature resistance of DCA microspheres}

Temperature resistance of microspheres was essential for the persistence of profile control and water shutoff. At a heating rate of 
Citation: Changchun Y, Xiangan Y, Daiyu Z, Rui X, Ji Z, et al. (2016) Feasibility Study on the Microspheres for Deep Profile Control Technology in High Permeability of High Temperature and High Salinity Reservoirs. J Pet Environ Biotechnol 7: 296. doi: 10.4172/2157-7463.1000296

Page 3 of 5

$10^{\circ} \mathrm{C}$ per minute, the temperature performance of DCA microspheres and hydrolyzed polyacrylamide (HPAM) were measured in an oxygen environment by thermal gravimetric analyzer (TGA) (Figures 3 and 4).

According to the heat gravimetric analysis results, the temperature resistance of DCA microspheres was characterized by a temperature point and at this point the slope of this stage's tangent is the maximum.

Figures 3 and 4 showed that temperature capability of DCA microspheres was up to $300^{\circ} \mathrm{C}$. Weight loss of DCA microspheres occurred in three phases which was from $50^{\circ} \mathrm{C}$ to $100^{\circ} \mathrm{C}$, from $260^{\circ} \mathrm{C}$ to $320^{\circ} \mathrm{C}$, and from $330^{\circ} \mathrm{C}$ to $460^{\circ} \mathrm{C}$. The weight loss of the first phase was mainly caused by the presence of a small amount of oligomers of the surface of DCA microspheres. A small amount of oligomers of the length of critical precipitation chain was longer, so its were unable to closely crosslink with DCA microspheres. Overall, weight loss of DCA microspheres occurred mainly in the second phase. Weight loss of the second phase was about $70 \%$. The proportion of weight loss was not too much in the first and third phase. Weight loss was less than $10 \%$ in the first phase and was about $20 \%$ in the third phase. Therefore, comprehensive analysis showed that weight loss of the core of DCA microspheres occurred in the third phase. Because the formation of the microspheres need to go through forming the phase of the core firstly and forming the phase of the ball later. The core was formed by the

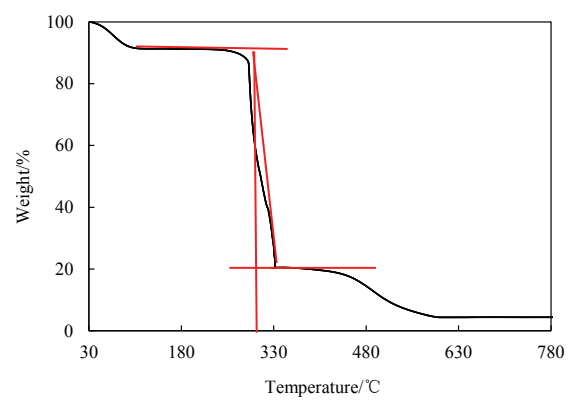

Figure 4: TGA result of DCA microspheres

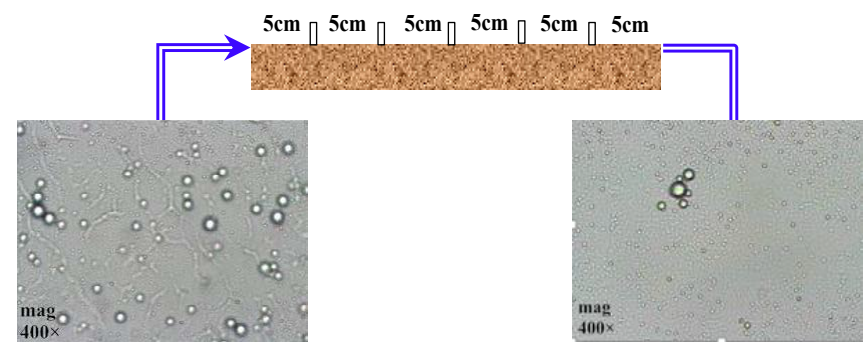

Figure 5: Microstructure of DCA microspheres in injection original liquid and recovery solution.

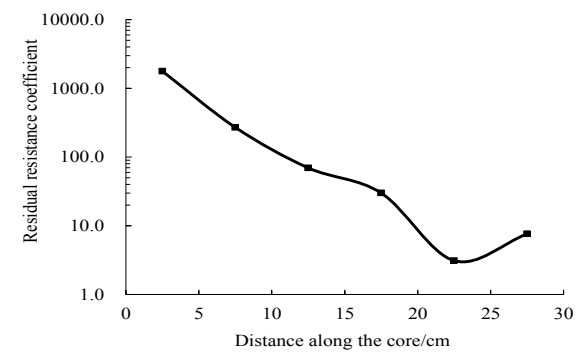

Figure 6: Residual resistance coefficient change with the distance along the core precipitation and aggregation of oligomer which was mainly composed of the structural units of divinylbenzene, so temperature resistance of the core was the strongest. The weight loss of the sphere which was between the surface of DCA microspheres and the core occurred in the second phase. The initial temperature of weight loss in the second phase characterize temperature resistance of DCA microspheres.

The temperature capability of HPAM and DCA microspheres were $193^{\circ} \mathrm{C}$ and $300^{\circ} \mathrm{C}$ respectively. The temperature capability of DCA microspheres was higher than profile control and water shutoff of conventional HPAM above $107^{\circ} \mathrm{C}$. According to relationship between viscosity and temperature, HPAM adapted to reservoir temperature from $70^{\circ} \mathrm{C}$ to $80^{\circ} \mathrm{C}$. According to decomposition temperature, DCA microspheres adapted to the target reservoir temperature above $130^{\circ} \mathrm{C}$.

\section{Plugging ability of DCA microspheres}

In order to determine the plugging ability of DCA microspheres to the large pores and cracks, the injection and plugging ability was evaluated by artificial homogeneous columnar core which had a diameter of $2.5 \mathrm{~cm}$ and a length of $30 \mathrm{~cm}$ at $115^{\circ} \mathrm{C}$. Gas permeability was $1.4 \mu \mathrm{m}^{2}$ to simulate water channel of actual reservoir. The pressure distribution along the cylindrical core was monitored in real time. The diameter distribution of the DCA microspheres in this test was shown in Figure 2. The concentration of the microspheres was $1000 \mathrm{mg} / \mathrm{L}$ at $115^{\circ} \mathrm{C}$. The microstructure of DCA microspheres was shown in Figure 5 in injection original liquid and recovery solution.

The plugging ability of DCA microspheres was characterized by residual resistance coefficient were calculated by the using of the Eqs. (1) . The result of evaluating the plugging ability of DCA microspheres was shown in Figure 6.

$$
R_{k}=\frac{K_{3}}{K_{1}}=\frac{Q \mu L / A \Delta p_{3}}{Q \mu L / A \Delta p_{1}}=\frac{\Delta p_{1}}{\Delta p_{3}}
$$

Where $R_{k}$ is residual resistance coefficient, $K_{1}$ is permeability of the core before injecting microspheres, $K_{3}$ is permeability of the core after injecting microspheres, $\Delta p_{1}$ is differential pressure of adjacent measurement points when the permeability of the core is measured in the stage of subsequent water flooding, $Q$ is flowing rate of the fluid through the core, $A$ is cross-sectional area of the core, $L$ is the length of the core, $\mu$ is the viscosity of the fluid through the core.

As shown in Figure 6, the residual resistance coefficient along the cylindrical core was greater than 2 . The highest value of residual resistance coefficient was 1777 . The microspheres have a stable plugging ability to migrate into all the measure points of the core to achieve the deep plugging. Hence, the DCA microspheres show high-strength deep plugging in reservoirs and are used as deep profile control agent of the high temperature and high salinity reservoirs.

\section{Effect of profile control and water shutoff of DCA microspheres}

The temperature and pressure of the experiment were at $115^{\circ} \mathrm{C}$ and $20 \mathrm{MPa}$. The plugging effect of the DCA microsphere was evaluated using two layers of the longitudinal heterogeneous artificial core (Figure 7). The length, width and height of the core were $30 \mathrm{~cm}, 4.5$ $\mathrm{cm}$, and $4.5 \mathrm{~cm}$ respectively. The distribution of the longitudinal gas measured permeability of the region of low permeability and high permeability was $0.22 \mu \mathrm{m}^{2}$ and $1.4 \mu \mathrm{m}^{2}$. The thickness of each layer were $3.5 \mathrm{~cm}$ and $1.5 \mathrm{~cm}$ respctively. The effect of profile control and water shutoff of DCA microspheres was shown in Figure 8. The appearance of produced liquid of subsequent water flooding was shown in Figure 9. 
Citation: Changchun Y, Xiangan Y, Daiyu Z, Rui X, Ji Z, et al. (2016) Feasibility Study on the Microspheres for Deep Profile Control Technology in High Permeability of High Temperature and High Salinity Reservoirs. J Pet Environ Biotechnol 7: 296. doi: 10.4172/2157-7463.1000296

Page 4 of 5

Figures 8 and 9 shows that the rate of enhanced oil recovery reached $56 \%$ in the stage of water flooding and was close to oil recovery of water flooding of Tarim oilfield which ranged from $50 \%$ to $55 \%$. The rate of enhanced oil recovery reached $10.18 \%$ and the water cut dropped by $20.73 \%$ after injecting only the slug of the DCA microspheres. The DCA microspheres flowed into the high-permeability layer, preferentially at $1.4 \mu \mathrm{m}^{2}$. The chain of the acrylamide molecule of the microsphere surface is bridged and blocked in the pore constriction through a covalent bond. The shell of the microspheres has pores in the size of nanometers. These shells slightly swell upon contact with water and also produce flow resistance. The injected water subsequently flows into the low-permeability layers $\left(0.22 \mu \mathrm{m}^{2}\right)$, which are not involved in starting the residual oil. Thus, the subsequent injection of fluid forces the occurrence of flow steering. The low-permeability region of the model was a white color inside yellow circle in Figure 7b. It further illustrated that the DCA microspheres adapt well to deep profile control and water shutoff in high permeability of the high-temperature and high-salinity reservoirs. The remaining oil of the unswept regional, whose relative permeability was lower, was started.

\section{Method optimization of effect of profile control and water shutoff of DCA microspheres}

To further optimize the method of effect of profile control and water shutoff of DCA microspheres, the experimental conditions was the exactly same with the above section. At the end of subsequent

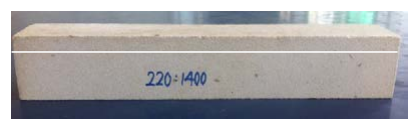

a) model appearance before saturated oil

Figure 7: Model appearance of two layers of the longitudinal heterogeneous artificial core.

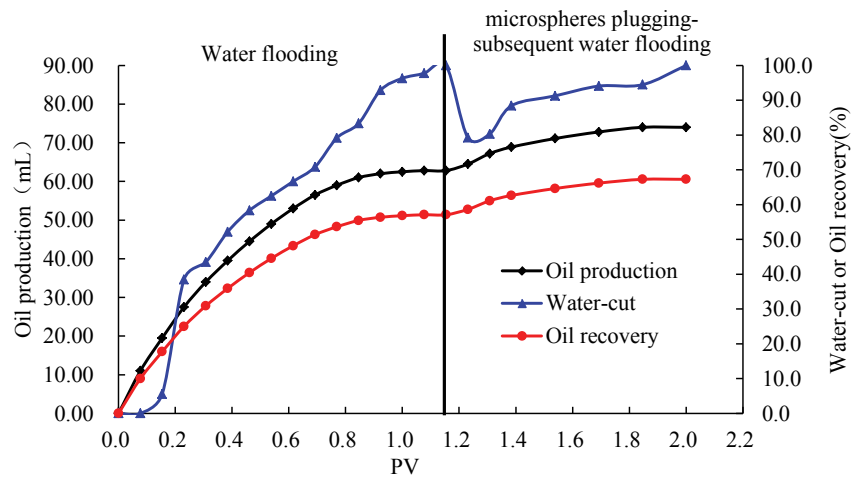

Figure 8: The effect of profile control and water shutoff of DCA microspheres.

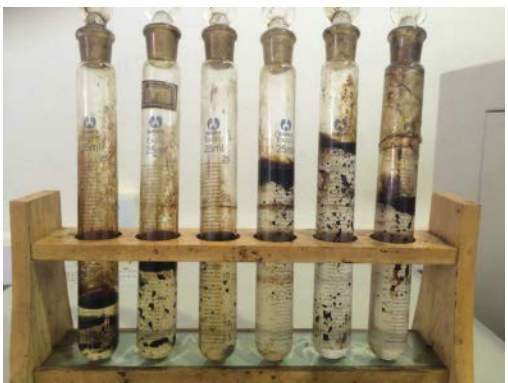

Figure 9: The appearance of produced liquid of subsequent water flooding

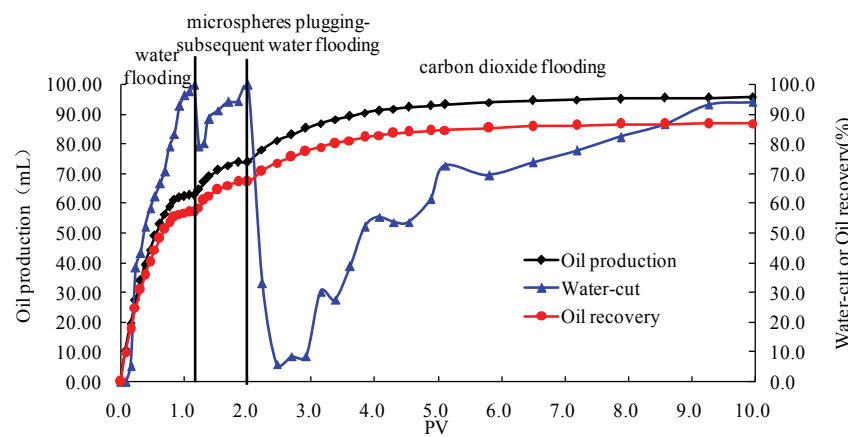

Figure 10: Method optimization of the effect of profile control and water shutoff of DCA microspheres.
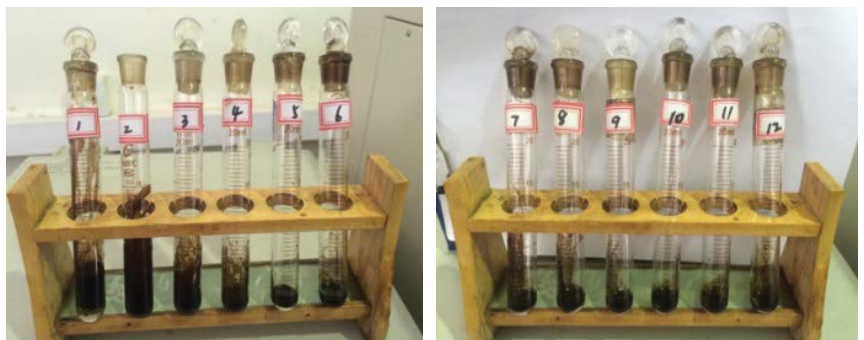

Figure 11: The appearance of produced liquid of carbon dioxide flooding

water flooding, the further method was carbon dioxide flooding by the longitudinal heterogeneous artificial core. The effect was shown in Figure 10. The appearance of produced liquid of carbon dioxide flooding was shown in Figure 11.

The rate of enhanced oil recovery reached $10.18 \%$ after injecting only the slug of the DCA microspheres in Figure 10. After subsequent water flooding, the further method was carbon dioxide flooding and the rate of enhanced oil recovery reached $19.64 \%$.

The DCA microspheres flowed into the high-permeability layer, preferentially at $1.4 \mu \mathrm{m}^{2}$. The chain of the acrylamide molecule of the microsphere surface was bridged and blocked in the pore constriction through a covalent bond. The shell of the microspheres had pores in the size of nanometers. These shells slightly swelled upon contact with water and also produced flow resistance. The injected carbon dioxide flows into the low-permeability layers $\left(0.22 \mu \mathrm{m}^{2}\right)$, which are not involved in starting the residual oil. The crude displacement with carbon dioxide enhanced oil recovery and one important mechanism of carbon dioxide emulsified crude oil. The produced liquid can see the obvious appearance of emulsification (Figure 11). The mechanism of displacing oil with carbon dioxide also includes volume expansion of crude oil, reducing viscosity of crude oil, changing the wettability of the rock, acidizing plugging removal, improving oil-water mobility ratio, reducing the interfacial tension, forming gas block phenomenon, extraction and vaporization, solution gas drive.

\section{Conclusion}

DCA microsphere had a three-layer structure and have better suspension. The temperature capability of DCA microspheres was up to $300^{\circ} \mathrm{C}$ and had better temperature resistance. The residual resistance coefficient along the cylindrical core was greater than 2 . The microspheres have a stable plugging ability to migrate into all the measure points of the core to achieve the deep plugging. DCA microsphere give raise in increase of oil production in oil wells, while 
Citation: Changchun Y, Xiangan Y, Daiyu Z, Rui X, Ji Z, et al. (2016) Feasibility Study on the Microspheres for Deep Profile Control Technology in High Permeability of High Temperature and High Salinity Reservoirs. J Pet Environ Biotechnol 7: 296. doi: 10.4172/2157-7463.1000296

Page 5 of 5

the water content in produced oil is decreased. At the end of water flooding, the slug of the DCA microspheres was only injected by the longitudinal heterogeneous artificial core. The rate of enhanced oil recovery reached $10.18 \%$ in stage of the subsequent water flooding. On the basis of the subsequent water flooding, the rate of enhanced oil recovery reached $19.64 \%$ in stage of carbon dioxide flooding. Feasibility methods which was the plugging of DCA microspheres and carbon dioxide flooding on deep profile control technology in high permeability of high temperature and high salinity reservoirs have been proposed.

\section{Acknowledgement}

This research was financially supported by National Key Scientific and Technological Project (Grant No.2011ZX05009-004) and National Basic Research Program of China (973Program) (Grant No.2011CB707305)

\section{References}

1. Wang $\mathrm{YH}$ (2014) Preparation and flocculating performance of inverse emulsion of water soluble polymer. Xi'an Shiyou University Press.

2. Zhu LP (2004) Preparation of functional polymer microsphere by inverse suspension polymerization. Tianjin University Press.

3. Bu DL (2013) Investigation on polymer microsphere profile control agent by dispersion copolymerization. Chengdu University of Technology Press.

4. Zhang YH, Dai CL, Ji WJ, Wang SY, Yang S, et al. (2012) Profile control and flooding mechanism and application methods of polymer microsphere. Chemical Engineering of Oil \& Gas 41: 508-540.

5. Lin WM, Chen YH, Cao M, Dong JY (2011) Evaluation and application of YG polymer microspheres as deep profile control agent. Oilfield Chemistry 28 : 327-330.

6. Sun $\mathrm{HQ}$, Wang $\mathrm{T}$, Xiao JH, Chen $\mathrm{H}$ (2006) Novel technique of in-depth profile control step by step by polymer microspheres. Petroleum Geology and Recovery Efficiency 13: 77-79.

7. He J, Yue XA, Sun Y, Feng XG, Tan X (2015) Preparation of uniform poly (acrylamide -co- DVB) microspheres in a low toxicity solvent by dispersion polymerization. Australian Journal of Chemistry 68: 1276-1281.

8. He X, Wu WM, Li L, Liu GY (2012) Application of polymer microspheres in water plugging of clastic rock horizontal wells with high temperature and high salinity. Complex Hydrocarbon Reservoirs 5: 82-84.

9. Vasquez J, Civan F, Shaw TM, Dalrymple ED, Eoff L, et al. (2003) Laboratory evaluation of high-temperature conformance polymer systems. SPE Production and Operations Symposium, SPE-80904-MS, Society of Petroleum Engineers, Oklahoma City, Oklahoma, USA.

10. Tang XF, Liu YZ, Qin H, Cai L, Jiang RY (2004) A new method of in-depth profile modification for high-temperature and high-salinity reservoir. SPE Asia Pacific Oil and Gas Conference and Exhibition, SPE-88468-MS, Perth, Western Australia, Australia.

11. Yang CC, Yue XA, Zhou DY, He J, Zhao J, et al. (2016) Performance evaluation of polymer microsphere with high temperature resistance and high salinity tolerance. Oilfield Chemistry 33: 254-260. 